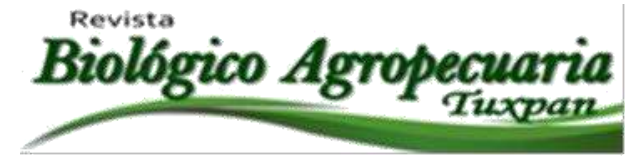

\title{
Calidad bromatológica y fisicoquímica de Moringa (Moringa oleifera Lam) producidas en la zona
}

\section{centro de Veracruz}

Bromatological and physicochemical quality of Moringa (Moringa oleifera Lam) produced in the central zone of Veracruz

Dávila Lezama, María del Rosario ${ }^{1 凶}$; Ramírez Hernández Teresita ${ }^{1}$; Rojas Avelizapa Luz Irene ${ }^{1}$; Juárez Juárez María Antonieta Rocío ${ }^{1}$ y Enríquez Ruvalcaba Vidal ${ }^{1}$

${ }^{1}$ Universidad Veracruzana. Facultad de Ciencias Biológicas y Agropecuarias campus Córdoba.

${ }^{凶}$ Autor para correspondencia: $\underline{\text { rdavila@uv.mx }}$

Recibido: $25 / 09 / 2017$

Aceptado: 18/12/2017

\section{RESUMEN}

Moringa oleifera Lam es un árbol originario del Himalaya, desde el noreste de Pakistán hasta el norte de Bengala del oeste en la India, es conocido como el árbol milagroso o el árbol de la vida. Se evaluó la calidad bromatológica y fisicoquímica de las hojas en fresco y deshidratadas de dos municipios de la zona centro del Estado de Veracruz, Tierra Blanca y Tepatlaxco incluyendo un análisis edafológico del suelo de las dos zonas. Los resultados obtenidos con respecto a los bromatológicos fueron: La moringa de Tierra Blanca reportó alto contenido de proteínas $23.62 \%$ con respecto a la moringa de Tepatlaxco $1.75 \%$ y la comercial $8.31 \%$, de cenizas la moringa de Tepatlaxco reportó un $3.81 \%$, contenido mayor que la de Tierra Blanca 3.69\% y menor con la comercial 6.92\%, de fibra la moringa de Tierra Blanca reporto valores cercanos a la comercial con $16.19 \%$ y $15.24 \%$, para el contenido de grasa se obtuvo valores de $2.87 \%, 2.36 \%$ y $3.40 \%$ en Tepatlaxco, Tierra Blanca y comercial respectivamente. los análisis fisicoquímicos los resultados de $\mathrm{pH}$ de $5.85 \%$ acidez de $12.8 \%$ y grados brix de $0.9 \%$ de la moringa de Tepatlaxco reporta valores que son sugeridos para un manejo poscosecha de conservación: deshidratación. El análisis edafológico reportó que el suelo de Tepatlaxco tiene un contenido de materia orgánica de $8.56 \%$ y un suelo arcilloso que favorece la retención de agua sugerido para el cultivo de la moringa. Se concluye que la moringa puede ser alternativa de una alimento funcional y se recomienda determinar los contenidos de algunos minerales que reportan contiene la moringa producida en las zonas de estudio.

Palabras clave: Moringa, Propiedades, Características.

\begin{abstract}
ABSTRAC
Moringa oleifera Lam is a tree native to the Himalayas, from the northeast of Pakistan to the north of West Bengal in India, it is known as the miraculous tree or the tree of life. The bromatological and physicochemical quality of the fresh and dehydrated leaves of two municipalities of the central zone of the State of Veracruz, Tierra Blanca and Tepatlaxco was evaluated, including an edaphological soil analysis of the two zones. The results obtained with respect to the bromatological ones were: The
\end{abstract}


moringa of Tierra Blanca reported high protein content $23.62 \%$ with respect to the moringa of Tepatlaxco $1.75 \%$ and the commercial $8.31 \%$, of ashes the Moringa of Tepatlaxco reported a $3.81 \%$, higher content that the Tierra Blanca $3.69 \%$ and lower with the $6.92 \%$ commercial fiber, the moringa of Tierra Blanca reported values close to the commercial with $16.19 \%$ and $15.24 \%$, for the fat content was obtained values of $2.87 \%, 2.36 \%$ and $3.40 \%$ in Tepatlaxco, Tierra Blanca and commercial respectively. the physicochemical analysis the $\mathrm{pH}$ results of $5.85 \%$ acidity of $12.8 \%$ and degrees brix of $0.9 \%$ of the moringa of Tepatlaxco report values that are suggested for a postharvest conservation management: dehydration. The edaphological analysis reported that the soil of Tepatlaxco has an organic matter content of $8.56 \%$ and a clayey soil that favors the retention of water suggested for the cultivation of moringa. It is concluded that moringa can be an alternative to a functional food and it is recommended to determine the contents of some minerals that report contains the moringa produced in the study areas.

Key words: Moringa, Properties, Characteristics.

\section{INTRODUCCIÓN}

Moringa es un árbol originario del sur del Himalaya, que se ha extendido a otras partes de India, Bangladesh, Afganistán, Pakistán, Sri Lanka, Sudeste Asiático, Asia Occidental, Península Arábica, África del Este y del Oeste, Sur de la Florida, Caribe, Centroamérica y gran parte de América del Sur. En América tropical se cultiva generalmente como planta ornamental, se cree que fue llevada de la India a África por los ingleses e introducida al Caribe por los franceses y de allí a Centroamérica (Vivien, 1990). Las flores son ricas en calcio y potasio, pueden consumirse crudas o cocinadas, acompañando ensaladas, sopas, otros platos y como infusiones. De las semillas se extrae un aceite similar al de oliva, muy bueno para el aliño de ensaladas. Estas, tiernas y hervidas en agua, son similares a los garbanzos; secas y tostadas, recuerdan al maní. Por su parte, el fruto es una vaina o cápsula triangular, ampliamente consumida en forma de guisos, es famoso por sus propiedades afrodisíacas, rico en proteínas, aminoácidos esenciales y múltiples vitaminas (Folleto de huertos familiares. Moringa. Cuba; 2011). Contiene altos niveles de caroteno (vitamina A), vitaminas B1, B2, B3, C, E, K, además de calcio, hierro, potasio, cobre, magnesio, zinc, todos los aminoácidos esenciales y antioxidantes (ácido ascórbico, flavonoides, fenoles, carotenoides, entre otros).

La moringa también es rica en ramnosa, un azúcar simple, glucosinatos e isotiocianatos. Sus raíces contienen moringina y moringininea, además de otros compuestos, tales como: fitosterol, ceras, resinas, zeatina, quercetina, ácido cafeoilquínico, pterigospermina y kaempferol (Bonal et al., 2012). La moringa es el único género de la familia Moringaceae, éste comprende 13 especies, las cuales son árboles de climas tropicales y subtropicales; la especie más popular es la Moringa oleífera (Bonal et al., 2012). La familia Moringaceae es un grupo pequeño de plantas dentro del inmenso orden Brassicales, que incluye la familia de la col y del rábano, junto con la familia del mastuerzo y de las alcaparras (APG, 2009). La familia más cercanamente emparentada con Moringaceae es Caricaceae, la de la papaya, con la cual comparte la característica de presentar glándulas en el ápice del peciolo. La Moringa oleifera es una planta de origen tropical y se desarrolla en climas secos, semiáridos, semi-húmedos y húmedos. Crece bien en altitudes que van desde el nivel del mar hasta los 1,200 $\mathrm{m}$ y prospera en temperaturas altas, considerándose óptimas para un buen

Revista Científica Biológico Agropecuaria Tuxpan 5 (2) ISSN: 2007-6940 
comportamiento las que están entre 24 y $32^{\circ} \mathrm{C}$ (Reyes, 2004).

El crecimiento de las plantas jóvenes también se ve muy afectado por las condiciones de luz, sobre todo en los períodos más cálidos del año. Por lo que la siembra en una época no adecuada combinado con un riego irregular puede provocar consecuencias desastrosas, causando la pérdida del 20 al $30 \%$ de las plantas jóvenes, por corte, produciendo pérdida de material productivo (Duarte y Flores, 2004).

\section{MATERIALES Y MÉTODOS}

El estudio se inició con colectas de hojas frescas de moringa (Moringa oleífera Lam) en las dos zonas de estudio, también muestras de suelo en los municipios de Tierra Blanca y Tepatlaxco Veracruz. Obtenida la muestra de hoja de moringa (Moringa oleífera Lam) y la muestra del suelo, se llevaron al

Laboratorio del Área de Proyectos Agroindustriales de la Facultad de Ciencias Biológicas y Agropecuarias de la Universidad Veracruzana, campus Peñuela y el análisis de proteína se realizó en la Universidad Tecnológica del Centro de Veracruz. El material y reactivos para los análisis físicoquímicos, bromatológicos y edafológicos fueron proporcionados en el laboratorio de Agroindustrias y específicamente para la determinación de proteínas el material y el análisis se realizó en el Laboratorio de Bromatología de la Universidad Tecnológica de Veracruz (UTCV), campus Cuitláhuac, Veracruz. Se colectaron un total de 300 gr de hojas frescas de moringa (Figura 11) en Tierra Blanca Veraruz y 82 gr de hoja fresca de Tepatlaxco (Figura 12), a las cuales se les realizaron los análisis fisicoquímicos en estado fresco, determinación de humedad y bromatológicos. La determinación de humedad se realizó con estufa por la técnica de pérdida de peso, Se empleo una temperatura de $45^{\circ} \mathrm{C}$ y un tiempo de 3 horas. Se realizó sanitizado de las hojas de moringa, con hipoclorito de sodio a 5 ppm y una acidificación con ácido orgánico
(Ácido cítrico y/o ácido ascórbico) por un tiempo de 3 minutos. Se escurrieron eliminando la mayor cantidad de agua. Después del sanitizado, acidificado y eliminación de agua de las hojas de moringa, se procedió a la deshidratación, empleando un deshidratador marca Weston, modelo 74-1001-w (Figura 13) durante 3.0 horas con un tiempo $45^{\circ}$.

\section{RESULTADOS}

Se obtuvo que el valor de $\mathrm{pH}$ y el contenido de azúcares totales en la moringa en fresco de Tepatlaxco fue menor que los de la moringa de Tierra Blanca y el resultado de la acidez en las hojas de Tepatlaxco es mayor que el de Tierra Blanca, (Cuadro 7), como lo menciona Domene \& Segura. 2014, el pH, es la medida potenciométrica más importante $\mathrm{y}$ utilizada en la industria alimentaria y se puede considerar como la acidez activa, relacionado con los ácidos presentes y la presencia microbiana que permitirán periodos anaquel más largos y los grados Brix son indicadores en el sector de alimentos, para medir la cantidad aproximada de azúcares en vegetales, zumos de fruta, vino o líquidos procesados dentro de la industria agroalimentaria, ambos índices evaluados son considerados como índices de madurez y el contenido de acidez determina la concentración total de ácidos contenidos en un alimento, hortaliza o fruto. Se obtuvo que los contenidos de ceniza en las hojas de Tepatlaxco fue de $4.31 \%$ indicando un contenido mayor que en de Tierra Blanca con $3.65 \%$ (Cuadro 8), como mencionan Kirk et al. (1996), las cenizas de un alimento son un término analítico equivalente al residuo inorgánico que queda después de calcinar la materia orgánica e indican que la moringa contiene entre algunos elementos; cobre, magnesio, potasio, fósforo, calcio y zinc. Los resultados obtenidos de fibra en la moringa de Tierra Blanca con $16.58 \%$, son menores que los de Tepatlaxco con $29.80 \%$. Nielsen 
(1998), menciona que la fibra cruda es el residuo orgánico lavado y seco que resulta después de hervir sucesivamente la muestra con ácido sulfúrico e hidróxido de sodio diluido, esta fibra contiene principalmente celulosa, además de lignina y hemicelulosas.

En cuanto a grasa, Tepatlaxco contiene $2.68 \%$ de grasa y las hojas provenientes de Tierra Blanca contienen 3.18\%. Como lo indica Nielsen, (1998) los lípidos se definen como un grupo heterogéneo de compuestos que son insolubles en agua, pero solubles en disolventes orgánicos como éter, cloroformo o benceno. Así como también mencionan Aurand et al. (1987), todos los lípidos contienen carbono, hidrogeno y oxígeno, algunos también fosforo y nitrógeno siendo las hojas de Tierra Blanca las que contienen mayor concentración de estos elemento orgánicos. Siguiendo el método indicado por Panzo, (2012), se deshidrató la moringa determinando su rendimiento. Juárez citado por Panzo, (2012), indican que la deshidratación es un método de estabilización de alimentos basándose en la reducción de la actividad de agua, con la finalidad de disminuir el deterioro del alimento brindando estabilidad microbiana manteniendo sus características nutritivas.

La moringa de la zona de Tepatlaxco reportó resultados de $\mathrm{pH}$ cercanos a la moringa comercial con 5.85 y 5.9 respectivamente. El contenido de sólidos solubles en la moringa de Tepatlaxco fue de $1.05 \%$ valor aproximado en la moringa comercial con $0.95 \%$.

Los resultados del análisis bromatológico (Cuadro 9) de la moringa se obtuvieron en fresco y en deshidratado. El resultado de proteínas (Figura 18) que reportó el análisis indica que las hojas deshidratadas de Tierra Blanca con un porcentaje $23.62 \%$ y Tepatlaxco tiene un resultado de $1.75 \%$. Pérez y Zomora,
2002 mencionan que las proteínas son sustancias orgánicas que contienen carbono, hidrógeno, oxígeno y nitrógeno. Están compuestas de aminoácidos, algunos de los cuales son esenciales para nuestro organismo; es decir, que necesariamente han de ser ingeridos junto con la dieta, ya que nuestro cuerpo no es capaz de producirlos por sí solo.

Las cenizas reportaron que las cápsulas de moringa comercial reportaron más contenido de minerales $6.92 \%$ y las hojas deshidratadas de Tierra Blanca y Tepatlaxco muestran entre ambas una mínima diferencia de $.40 \%$ como indican Kirk et al 1996, la cantidad de cenizas resultantes de un alimento son equivalentes al residuo inorgánico que queda después de calcinar la materia orgánica.

Los resultados de fibra reportan que las hojas deshidratadas de Tepatlaxco contiene $21.74 \%$ y una diferencia de $.95 \%$ entre las hojas deshidratadas de Tierra Blanca y las cápsulas comerciales como menciona Kritchevsky 1988 la fibra cruda es el residuo orgánico combustible e insoluble que queda después de que la muestra es tratada con tratamientos consecutivos con éter de petróleo ligero, ácido sulfúrico diluido hirviente e hidróxido de sodio hirviente, este tratamiento proporciona el contenido en celulosa además de la lignina y hemicelulosas.

Los resultados de grasa resultaron que las capsulas comerciales de moringa fueron mas altas que Tierra Blanca y Tepatlaxco en porcentaje con $1.04 \%$ y 0.53 respectivamente según Aurand et al 1987 todos los lípidos contienen carbón, hidrógeno y oxígeno, y algunos también contienen fósforo y nitrógeno. 
Figura 1. Grados Brix en hojas frescas y deshidratadas de Moringa de Tierra Blanca y

Tepatlaxco y capsulas comercial Veracruz.

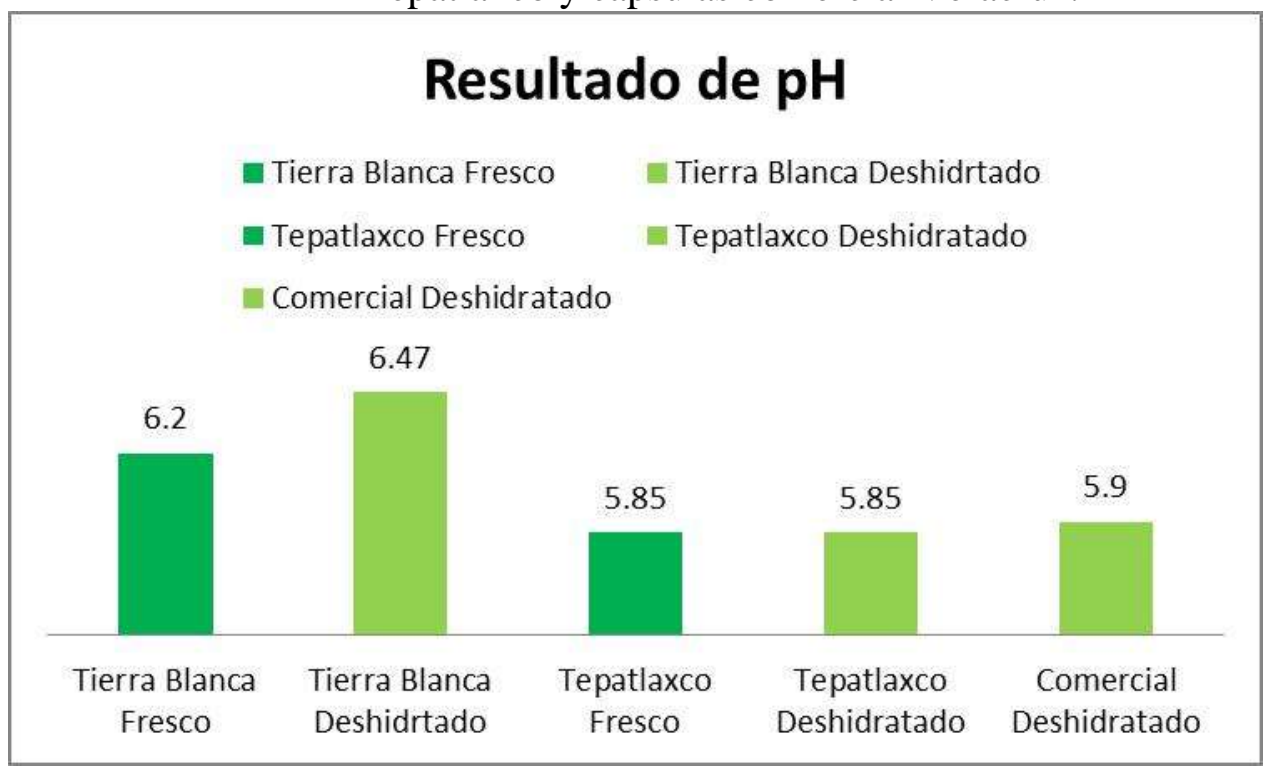

Cuadro 1. Resultados de los análisis bromatológicos en hojas deshidratadas de Moringa.

\begin{tabular}{|l|l|l|l|l|}
\hline Tratamiento & \%Proteínas & \% Cenizas & \% Grasa & \% de Fibra \\
\hline Tierra Blanca & 23.62 & 3.69 & 2.36 & 16.19 \\
\hline Tepatlaxco & 1.75 & 3.82 & 2.87 & 21.74 \\
\hline Comercial & 8.31 & 6.92 & 3.40 & 15.24 \\
\hline
\end{tabular}

El suelo colectado de Tierra Blanca y Tepatlaxco, el análisis edafológico realizado reportó.

De acuerdo a los resultados se obtuvo que el $\mathrm{pH}$ del suelo de Tierra Blanca es mayor al de Tepatlaxco, esto indica que el del suelo de
Tepatlaxco es moderadamente ácido y el de Tierra Blanca es neutro, como mencionan Parra et al 2002 la importancia de medir el pH de un suelo radica en la disponibilidad de los nutrientes del suelo por parte de las plantas para absorberlos, la lectura del $\mathrm{pH}$ se refiere a la concentración de los componentes sólidos y líquidos. 


\section{CONCLUSIÓN}

Los análisis fisicoquímicos, de $\mathrm{pH}$ y grados brix, de las cápsulas comerciales de Moringa tiene casi los mismos resultados que las hojas frescas y deshidratadas de Tepatlaxco, en acidez resultó que para las hojas deshidratadas aumento el nivel de acidez siendo las muestras de Tierra Blanca con más alto nivel y las comerciales en menor cantidad de acidez. Entre los usos de la moringa es el consumo en fresco y empleando el método de conservación por deshidratación la moringa de Tepatlaxco es en la que se obtuvo menor $\mathrm{pH}$, variable importante en métodos de conservación de alimentos.

\section{LITERATURA CITADA}

Abdulkarim S., Long, Lai O., Muhammad S. Ghazali H. (2005).Some physicoquemichal properties of Moringa Oleifera sid oil extracted using solvent and aqueous enzymathic metods. Food chemistry 93,253-263. https://doi.org/10.1016/j.foodchem.2004.09.

CONACYT: Consejo Nacional de Ciencia y Tecnología (2008). Rendimiento y Uso Potencial de Paraíso Blanco, Moringa oleífera Lam en la Producción de Alimentos de Alto Valor Nutritivo para su Utilización en Comunidades de Alta Vulnerabilidad Alimentario-nutricional de Guatemala.

Alfaro, N. C., Martínez, W. (2008). Uso Potencial de la Moringa (Moringa oleífera, Lam) para la Producción de Alimentos Nutricionalmente Mejorados, Consejo Nacional de Ciencia y Tecnología (CONCYT), Secretaría Nacional de Ciencia y Tecnología (SENACYT), Fondo Nacional de Ciencia y Tecnología (FONACYT), Instituto de Nutrición de Centro América y Panamá (INCAP), Guatemala.

Arias Jiménez A, C.(2007). Suelos tropicales. universidad estatal a distancia, san José, costa rica, $188 \mathrm{p}$.
Cáceres, Armando, (1993) Actividad antiinflamatoria de plantas medicinales de uso popular en Guatemala (I). Guatemala: DIGI, USAC, $61 \mathrm{p}$.

Castellanos, J. Z., Uvalde - Bueno, J. X., y Aguilar Santelises, A. (2000). Manual de interpretación de Análisis de Suelos y Aguas (2a. Edición). Universidad Autónoma Chapingo, México.

Duarte, F. J., Flores Leiva, B. A. (2004). Producción de Biomasa de Moringa oleífera sometida a diferentes densidades de siembra y frecuencia de corte, en el trópico seco de Managua, Nicaragua, Universidad Nacional Agraria, Facultad de Ciencia Animal, Managua, Nicaragua

Guzman Luis (2012). Moringa oleífera "la planta de los mil usos" revista virtual: Moringa

Dominicana disponible en : http://moringadom.blogspot.mx/ citado 20 junio 2016

Lopez, S. (2009). Moringa oleifera. Consultado el 21 de Junio de 2016. Disponible en:

Perez Llamas Francis, Zamora Navarro Salvador (2002). Nutrición y alimentación humana.

Editorial Servicio de Publicaciones

Universidad de Murcia 1ra. Edicion

Terence D, pennington. Sarukhan J .(2005). arboles tropicales de México. Manual para la identificación de las principales especies. Texto científico universitario, México, 523 p. Disponible en

Villaseñor R., J. L. y F. J. Espinosa G. (1998). Catálogo de malezas de México. Universidad Nacional Autónoma de México, México, D. F. $448 \mathrm{p}$.

Yanaka, A., S. Zhang, M. Yamamoto y J. W. Fahey. (2005). Daily intake of sulforaphane-rich broccoli sprouts improves gastritis in $\mathrm{H}$. pylori-infected human subjects. Cancer Epidemiology Biomarkers and Prevention 14: $14: 2754 s$ 
Copyright (c) 2017 Maria del Rosario Dávila Lezama, Teresita Ramirez Hernández, Luz Irene Rojas Avelizạa,

Maria Antonieta Rocio Juárez Juárez y Vidal Enriquez Ruvalcaba

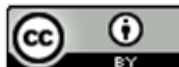

Este tex to está protegido por una licencia licencia Creative Commons 4.0

Usted es libre para Compartir —copiar y redistribuir el material en cualquier medio o form ato-y Adaptar el documento —remezclar, transformar y crear a partir del material- para cualquier propósito, , incluso para fines comerciales, siempre que cumpla la condición de:

Atribución: Usted debe dar crédito a la obra original de manera adecuada, proporcionar un enlace a la licencia, e in dicar si se han realizado cam bios. Puede hacerlo en cualquier form a razonable, pero no de form a tal que sugiera que tiene el apoyo del licenciante o 10 recibe por el uso que hace de la obra.

$\underline{\text { Resumendelicencia }}$ - Textocompletodelalicencia 